\title{
RUNNING THE RED LIGHT
}

\section{A trial drug encourages cells to ignore the signs that stop them making faulty proteins. Sound dangerous? Claire Ainsworth discovers that it could be a cure for genetic disease.}

n 28 November, two patients in an Israeli hospital downed a vanillaflavoured shake in the hope of curing their cystic fibrosis. The drug in the shake is meant to get their cells to ignore the genetic mutation that makes their lives a misery. Remarkably, if this trial works, the very same drug might be used to help patients with a huge range of genetic diseases.

At the moment treatments for cystic fibrosis concentrate on the condition's symptoms, such as lung-clogging mucus, rather than its underlying cause - a faulty gene. Advocates of gene therapy aim to correct this by delivering working copies of the cystic fibrosis gene to a patient's cells, but results have been mixed so far.

The drug in the shakes, PTC124, takes a different approach, concentrating neither on the gene itself nor on the symptoms it causes, but on the process that links the two. It is supposed to work on the system that translates genetic information into proteins, coaxing it into ignoring a specific sort of genetic defect. The drug cannot offer aid to all those with cystic fibrosis, because it is expected to help with only a particular subset of mutations. But if it works for a few people, it should also work against some of the other diseases in which single genes are damaged. It is estimated that one-third of people with an inherited genetic disease have the sort of mutations that PTC124 and similar treatments might get cells to ignore.

PTC124 is aimed at thwarting a cellular process called nonsense-mediated decay, or NMD. Research into this phenomenon has taken off only in the past ten years, but it is already offering potential therapies and insight

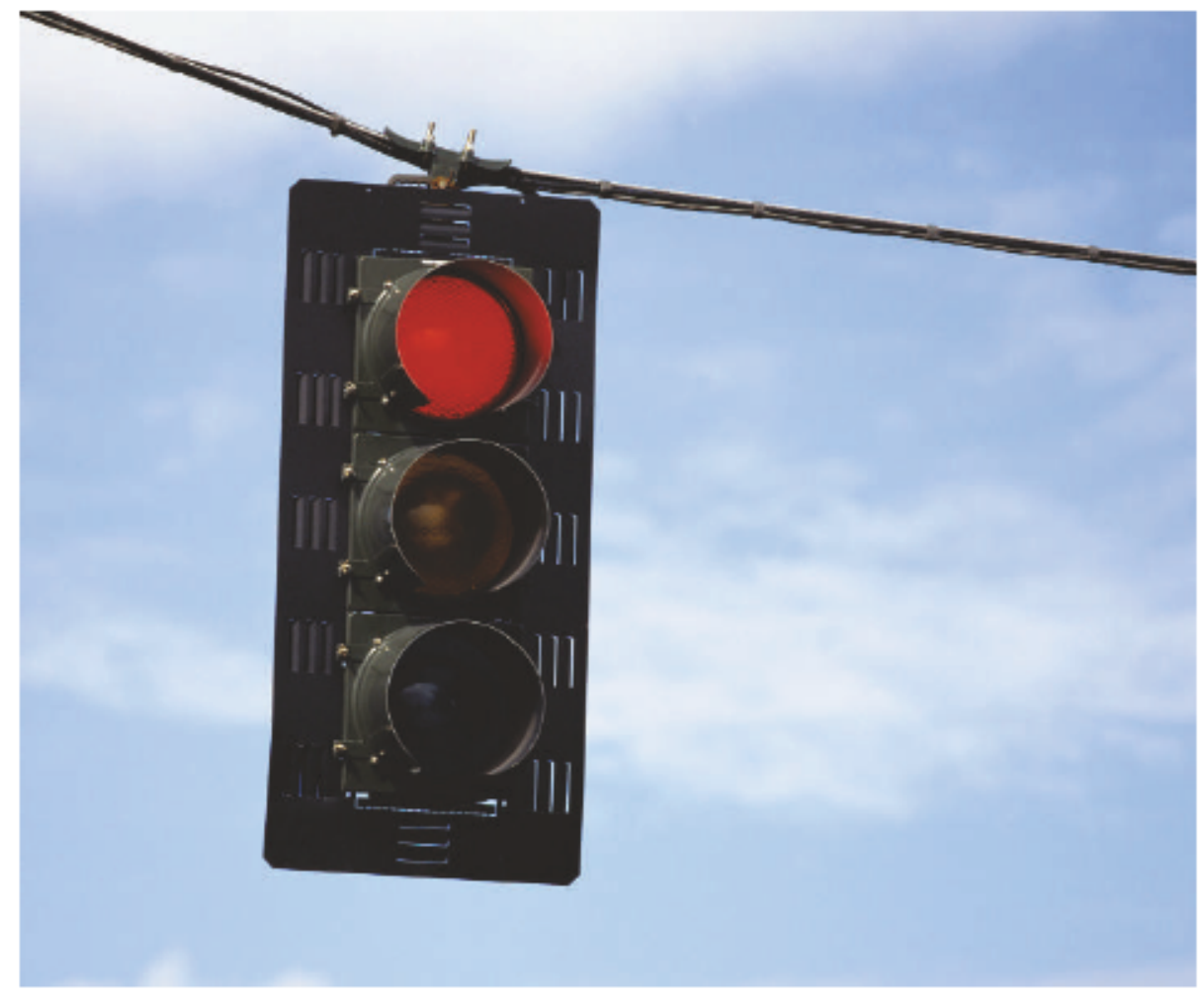

into the mysteries of information processing in the cell ${ }^{1}$. Some of the scientists involved hope that NMD will shed light on classic puzzles, such as why creatures with similar genetic make-up can have different physical characteristics and why our genome has evolved the way it has.

In the first step towards producing a protein from a gene, the cell makes a complementary RNA copy of the gene's DNA sequence. This copy, called messenger RNA, is packaged and processed and sent off to the cell's ribosomes - molecular machines that read RNA and put together proteins according to the instructions contained in the sequence. Each sequential group of three genetic letters, or codons, in the RNA tells the ribosome to add a particular amino acid to the protein that it is constructing. If the gene is damaged in some way, the RNA will be too. If a piece of DNA is missing, some codons will be lost; if it is garbled, the codons will be as well, and the resulting amino-acid chain may notbe a properly functioning protein.

But not all codons signify an amino acid. Some, the 'stop' codons, mark the end of the gene's protein-coding sequence; they tell the ribosome that the chain of amino acids it has been making should come to a close. Some mutations can cause a stop codon to appear in the middle of a messenger RNA, and it is these mutations that cause the NMD response.

In the late 1970s, scientists noticed something odd about a subset of patients with a potentially fatal inherited anaemia called $\beta$ thalassaemia. This disease affects $\beta$-globin, one of the subunits of haemoglobin, which carries oxygen in the blood. In some forms of the disease, patients make faulty $\beta$-globin; in others they don't seem to make the protein at all. Lynne Maquat, then a postdoc at the University of Wisconsin, Madison, was studying the bone marrow of patients that apparently made no $\beta$-globin and found that the messenger RNA for the protein disappeared unusually rapidly from their cells ${ }^{2}$. Maquat and others sequenced these patients' genes and found stop mutations in them. It seemed as if the extra stop codons were triggering a qualitycontrol process that disposed of the faulty messages. But no one could work out how a cell tells the difference between a normal stop codon and one caused by mutation.

\section{Don't shoot the messenger}

A key clue came from a set of patients with a rare form of $\beta$-thalassaemia. These patients made a shortened version of the protein - the version you would expect to see if the cell was allowed to read the faulty RNA and simply finished the protein when faced by a premature stop codon. The thing these rare cases had in common was that their extra stop codons all lay towards the end of the $\beta$-globin gene. Subsequent work by Maquat, now heading a team at the University of Rochester, New York, and by other groups has shown that the explanation lies in the way that messenger RNA is patched together from its gene ${ }^{3}$.

Not all the DNA in a human gene codes for amino acids. The bits of the gene that carry instructions for making the protein are called exons; the intervening bits are called introns. The introns are cut out of the RNA copy of the gene, and the remaining exons are spliced together. These splicing points have marker proteins stuck near them, like Post-it notes (see graphic). 


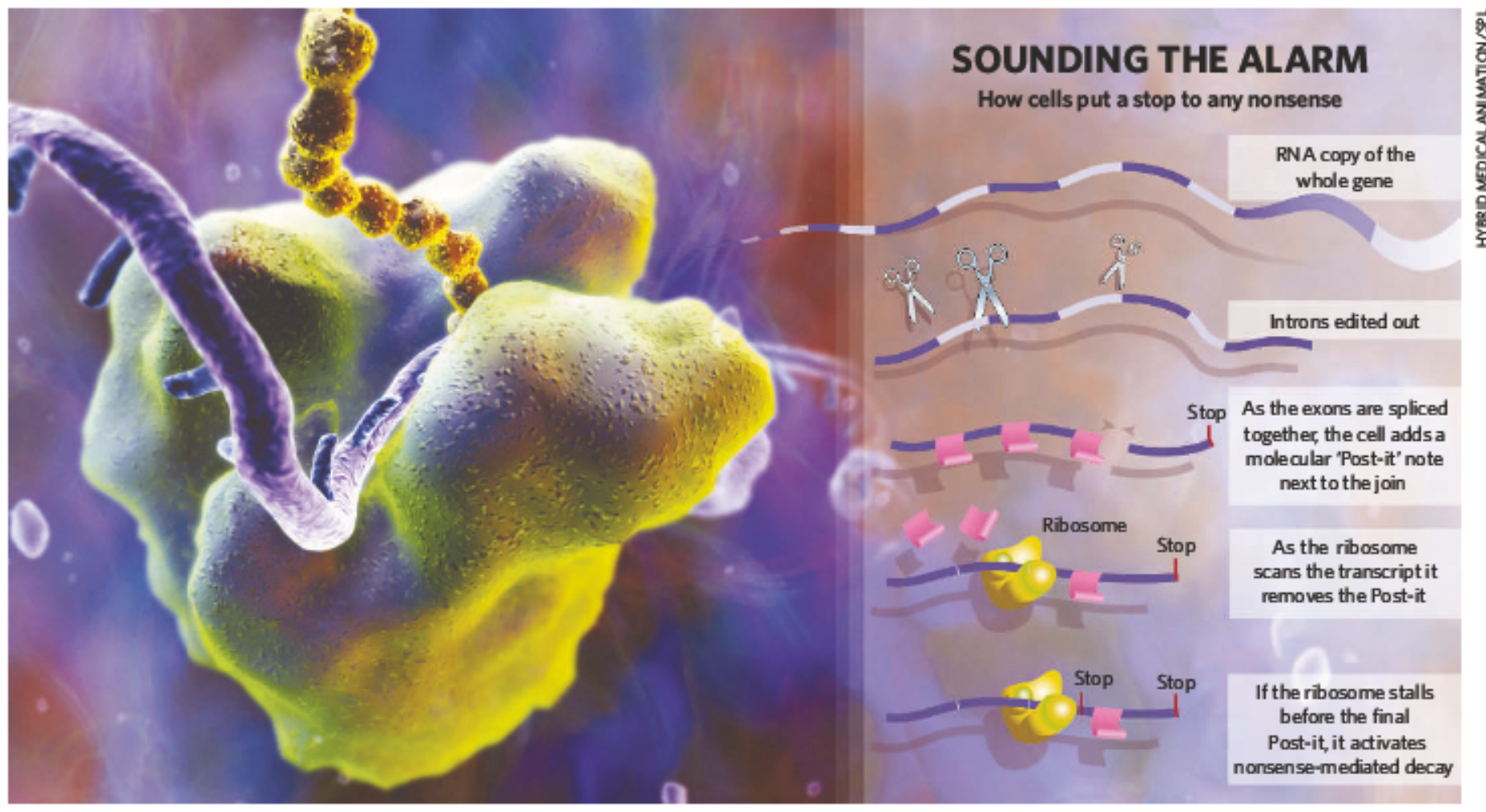

Researchers think these notes act as signposts, telling the ribosome where it is as it moves along the RNA, explains Elisa Izaurralde, who works on NMD at the European Molecular Biology Laboratory in Heidelberg, Germany. If the first ribosome that encounters the messenger RNA makes it past the final Post-it, the cell will be satisfied that the RNA is up to scratch, and allow other ribosomes to work on it. But if the scouting ribosome finds a stop codon before that point, alarm bells ring and the faulty message gets shredded by NMD. In the rare thalassaemia cases, the RNA survived to produce protein only because the stop codon appeared too late in the sequence to trigger the decay response.

The protein fragments that premature stop codons would produce if left to their own devices could do various types of damage; for example, they might stick to other proteins and stop them working properly. NMD gets rid of this problem. In some cases this limits the harm done by mutations with which we are born; in others it may lessen the effects of mutations we later acquire. The development of cancer often involves the appearance of stop codons in genes that would otherwise protect us from the disease, and it may be that the efficiency of a person's NMD response affects their predisposition to cancer. "It's an attractive speculation, and may well turn out to be true, but it is not yet proved," says Philip Anderson, a biologist who works on NMD in the Caenorhabditis elegans worm at the University of Wisconsin.

Still, NMD is a double-edged sword. In $\beta$-thalassaemia, shortened versions of the $\beta$ globin protein really would do damage, and it makes sense to silence the gene through NMD.
But shortened versions of the cystic fibrosis protein do not cause disease. "It's the other side of the coin," says Andreas Kulozik, a paediatrician at the University of Heidelberg who studies the decay response and its role in blood diseases. "If these truncated proteins were actually made, they would do some good." The same is probably true in many other conditions; not all protein fragments are harmful, and some help.

\section{Deliberate mistakes}

Researchers have not yet worked out how to control NMD. But what they can do is smuggle nonsense mutations under NMD's radar, and fool the cell into producing a protein that the decay response would prohibit. In a neat twist, they learned this trick from an antibiotic commonly used to treat the lung infections that plague cystic fibrosis patients.

Gentamicin belongs to a family of antibiotics called aminoglycosides that gum up the ribosomes of bacteria, making them prone to misreading messenger RNA. These befuddled ribsosomes will often mistake a stop codon for one specifying a run-of-the-mill amino acid and 'read through' it, adding that amino acid in its place. Researchers wondered whether they could pull off the same trick in human cells and so tiptoe past the NMD machinery. If the ribosomes were to read through instead of stalling, the decay process wouldn't come into play, and

"We used to call gentamicin

a magic drug, because some patients improved remarkably." - Eitan Kerem nearly normal proteins should be produced.

In 1997 a team at the University of Alabama, Birmingham, announced that it had managed to get human cells in culture to read through a faulty cystic fibrosis gene with the aid of gentamicin ${ }^{4}$. Two years later, a team led by Lee Sweeney of the University of Pennsylvania, Philadelphia, showed that the strategy could work in living animals ${ }^{5}$. Studying mice with nonsense mutations in the dystrophin gene, which is mutated in Duchenne muscular dystrophy, Sweeney's team found that those dosed with gentamicin produced full-length dystrophin protein, although much less of it than normal mice do. Movement did less damage to the muscles of treated mice than untreated mice.

The papers caught the eye of Eitan Kerem, a cystic fibrosis specialist at the Hadassah University Hospital in Jerusalem. $\mathrm{He}$ and his team had noticed that some of their patients with cystic fibrosis improved to an inexplicable extent when given gentamicin to inhale for lung infections, relapsing badly when the drug was withdrawn. "In some patients, we used to call it a magic drug, because they improved remarkably, he recalls. "We always said: there must be something else in this drug". And they were in a particularly good position to test the idea that gentamicin was suppressing NMD. In most populations, nonsense mutations are the cause of cystic fibrosis in just 2-5\% of cases. But owing to an ancient genetic accident and centuries of intermarriage, some $60 \%$ of Ashkenazi Jews with cystic fibrosis carry a nonsense mutation.

Kerem's team gave gentamicin nose drops to 19 cystic fibrosis patients who had stop-codon mutations. The drug restored protein function 
Clear the lungs: most treatments for cystic fibrosis simply tackle its symptoms.

\section{IMAGE UNAVAILABLE FOR COPYRIGHT REASONS}

it is also a rather messy one - likely to produce some RNAs with stop codons in them. A nonsense-mediated response reduces the risk that these stop codons will cause harm. And the interplay of NMD and the stop codons introduced by splicing may, in fact, be a way of controlling how and when genes get turned into proteins'. "As time goes on, there are more and more examples of 'purposeful' targets of NMD, says Anderson, citing his work on C. elegans. "These are not errors of gene expression, they are normal aspects of it."

Michael Lynch, a biologist at Indiana University in Bloomington, suggests that NMD may have helped to spread introns through genomes. Using a mathematical model, Lynch discovered that introns are far more evenly spaced in vertebrate genomes than would be expected by chance. Introns in organisms such as yeast and fruitflies, which do not seem to use the Post-it system to trigger NMD, are rarer or more randomly distributed. Lynch speculates that introns and the decay response scratched each other's backs through evolution: NMD meant that errors caused by multiplying introns were minimized, while introns that spaced themselves evenly in genes acted as regular signposts for ribosomes, making NMD work better. "But who was driving whom is really an open area of research," he says.

Anderson agrees that the decay response is a force to be reckoned with in evolution. By masking the effects of mutations that would otherwise reduce a creature's ability to survive, NMD gives genomes much greater freedom to experiment and evolve.

What is more, Anderson's team has shown that differing versions of the same gene behave differently, depending on the presence or absence of NMD machinery ${ }^{10}$. It is this idea that excites medics such as Hentze and Kulozik, who wonder whether differences in NMD could influence how badly people are affected by inherited diseases.

As well as saving lives, it seems that understanding NMD could help us to explain some of biology's biggest questions. But researchers still need hard data from molecular biology to make these theories stick. "It's easy to make these speculations, and very entertaining," says Anderson. "It's damned hard to prove, but to me it makes a lot of biological sense." Claire Ainsworth is asenior News and

\section{Features editor for Nature.}

1. Holbrook, J.A, Neu-Ylik, G. N, Hentze, M.W. \& Kulazik, A. E Nature Genet. 36, 801-808 (2004)

2. Maquat, L.E, Kinninburgh A. L, Rachmilewitz, E A.\& Ross, I Cell Z, 543-553 (1981).

3 Maquat, L.E.J. Cell Sci. 118,1773-1776 (2005)

4. Bedwell. D. M.et al. NatureMed 3,1280-1284 (1997).

5. Barton-Daviș E R, Cordlex, L, Shoturma, D.L, Leland, S. E. \&Sweeney, H.L.J. Clin Invest. 104, 375-381(1999).

6. Wilschanski, M.etal. N. Engl J.Med 349, 1433-1441 (2008)

7. Gehring N. H etal.Mol. Cell 20, 65-75(2005).

8. Wilkinson, M.F. Trends Genet. 21, 143-148 (2005)

9. Mendell, IT.et a. Nature Genet. 36, 1073-1078 (2004)

10. Cali, B. M.\& Anderson, P. Mal. Gen Genet. 260,176-184 (1998). to try it on other conditions, possibly induding

cancers in which nonsense mutations play a together in different ways lets cells make more than one protein from the same gene. This is a useful way to get the most out of a genome, but 